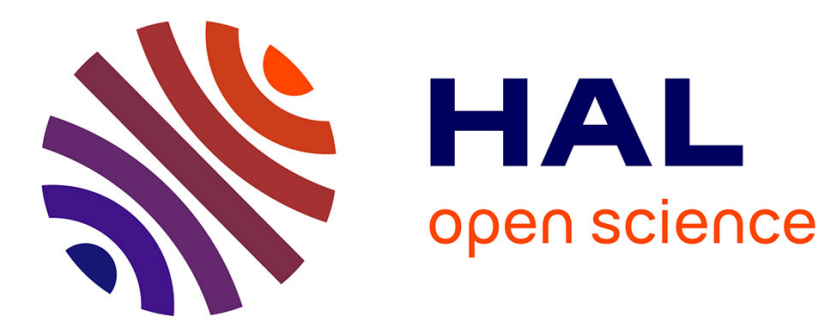

\title{
L'ABAISSEMENT DU TAUX DES ACIDES VOLATILS NE REND PAS LE BEURRE \\ "ANORMAL"
}

\author{
L. Hoton
}

\section{- To cite this version:}

L. Hoton. L'ABAISSEMENT DU TAUX DES ACIDES VOLATILS NE REND PAS LE BEURRE "ANORMAL". Le Lait, 1940, 20 (198_199_200), pp.497-510. hal-00927836

\section{HAL Id: hal-00927836 https://hal.science/hal-00927836}

Submitted on 1 Jan 1940

HAL is a multi-disciplinary open access archive for the deposit and dissemination of scientific research documents, whether they are published or not. The documents may come from teaching and research institutions in France or abroad, or from public or private research centers.
L'archive ouverte pluridisciplinaire HAL, est destinée au dépôt et à la diffusion de documents scientifiques de niveau recherche, publiés ou non, émanant des établissements d'enseignement et de recherche français ou étrangers, des laboratoires publics ou privés. 


\section{LE LAIT}

REVUE GEN ÉRALE DES QUESTIONS LAITIÈRES

\section{SOMMAIR E}

\section{Mémoires originaux :}

L. Hoton. - L'abaissement du taux des acides volatils ne rend pas le beurre "anormal"...... .

G. Génin. - L'utilisation du sérum . . . . . . .

J. Vieilly. - Le lait frais pasteurisé irradié à l'abri de l'air et la vitamine D. Indications médicales. Posologie ........

J. Lemorne. - Contribution à l'étude des emplois des aciers inoxydables dans les laiteries et les fromageries. . . . . . . .

ibliographie analytique :

10 Journaux, Revues, Sociétés savantes

$2 \circ$ Brevets

\section{Bulletin bibliographique :}

\section{Documents et informations :}

M. BEAU. - La situation laitière . . . . . . . . .

Loi du 27 juillet 1940 sur l'organisation de la produetion laitière . . . . .

Arrêté du 30 juillet 1940 nom. mant un Comité central des groupements interprofessionnels .......

Décret du 31 juillet 1940 portant restrictions sur le lait et les produits laitiers . . .

Arrêté du 20 août 1940 con. cernant le prix du lait et dérivés : beurre et fromages.

Décret du 26 août 1940 concernant l'organisation de la production laitière. . . 572

Décret du 15 novembre 1940. 591

Table des matières. . . . 593

Table des auteurs . . 599

Table des ouvrages analysés. 609

\section{MÉMOIRES ORIGINAUX (1)}

\section{L'ABAISSEMENT DU TAUX DES ACIDES VOLATILS NE REND PAS LE BEURRE " ANORMAL "}

par

\section{HOTON}

Docteur ès sciences

Le Dr Balavoine termine son étude sur le beurre anormal (Le Lait, décembre 1939) par cette considération très judicieuse : "Si regrettable que soit pour la sûreté du contrôle de la pureté du beurre le fait de l'abaissement, dans certaines conditions, de

(1) Reproduction interdite sans indication de source. 
l'indice R.M (acides gras volatils), il ne faut pas lui conférer une portée péjorative sur la qualité hygiénique de ceux quile présentent. »

Puis-je ajouter à cette considération très sensée, qu'à mon avis, les raisons données jusqu'ici pour justifier cette portée péjorative ne résistent pas à l'examen?

Nous nous trouvons devant le fait brutal de l'élévation et l'abaissement de l'indice A.V.S. à deux époques de l'année à peu près fixes : avril et novembre.

Pourquoi?

$$
* * *
$$

Je baserai mon argumentation non sur la composition des beurres d'étables ou de laiteries de moyenne importance, mais sur celle des beurres placés sous le contrôle de l'Etat hollandais, ces beurres sont analysés toutes les semaines ou tous les 15 jours; comme les quantités analysées vont de 75.000 kilogrammes à près de 1.000 .000 (Leewaarden), on peut fonder sur leurs résultats des conclusions sérieuses.

On s'est appuyé pour classer les beurres en normaux et anormaux (1) sur leur taux en acides volatils solubles (A.V.S.), qui caractérisent le groupe des acides butyriques caproïques, capryliques et capriques spécifiques du beurre. A l'époque (1887) où ReICHert et Meislu ont créé leur méthode de dosage, les experts n'avaient à leur disposition que des procédés permettant, il est vrai, de différencier les beurres des autres graisses; mais ces procẻdés étaient basés sur le dosage d'éléments communs à toutes les matières grasses alimentaires, tels l'indice d'iode, les indices Hehner, Kottstorfer, le procédé Dalican.

Les chimistes de ma génération crurent trouver dans le procédé Reichert-Meisll le moyen infaillible de déceler la falsification du beurre, puisqu'il permettait l'identification des éléments propres à celui-ci. On déclara que la norme " 26 A.V.S. » était, sans conteste, l'indice de la pureté du beurre, attendu que les matières grasses utilisées pour les mélanges, à cette époque, ne titraient que " 0,5 A.V.S. ». (La graisse du cocotier et des palmistes n'apparaîtra que 10 ou 12 ans après.)

Les chiffres A.V.S. fixés en 1888 étaient de 26 "minimum " et 33 " maximum ». L'axiome de cette époque était done que tout beurre titrant moins de 26 était falsifié! Quelques chimistes sont venus ultérieurement affirmer timidement que des beurres authentiques avaient donné un indice légèrement inférieur à 26 , on leur

(1) C'est à mon corps défendant que j'utilise ces termes dépourvus de toute valeur scientifique. 
répondait, et c'était aussi mon avis à cette époque : "Que cela ne pouvait résulter que d'une erreur d'analyse. »

Et l'on trouve dans la littérature de l'époque cette idée concrétiséé par VIrLers et Collin (Aliments lactés et Alimemts gras, Doin, 1911, p. 264) comme suit: " ou bien les beurres anormaux (inférieurs à 26) ont été fabriqués pour les besoins de la cause, ou bien les résultats (de l'analyse) ont été mal déterminés. ”

Les erreurs d'analyse pouvaient être invoquées à cette époque à cause du procédé de saponification à l'alcool remplacé aujourd'hui par la glycérine. Il fallut cependant se rendre à l'évidence : les travaux ultérieurs si complets du $\mathrm{D}^{\mathrm{r}}$ SWAVING en Hollande, ceux de VAN ENGelen et Pierre WAUters, de Jorissen et les miens étaient concluants : le chiffre A.V.S. était descendu à 25-24..., le $\mathrm{D}^{\mathrm{r}} \mathrm{SwA}-$ VING avait même constaté 20 .

$$
*^{*} *
$$

La première enquête officielle eut lieu en Belgique durant les années 1897-1898. Elle révéla l'existence de 10\% environ de beurres titrant moins que 26 A.V.S. Ses résultats furent contestés, car les chimistes s'étaient tous servis du procédé de saponification par l'alcool, les résultats pouvaient done différer d'opérateur à opérateur suivant le temps employé pour l'évaporation de l'alcool.

Un inspecteur du service central de la Répression des fraudes, M. E. Grognard, entreprit seul une seconde enquête dont les résultats ne furent jamais publiés.

Une troisième enquête fut poursuivie durant les années 19051906, elle portait sur 80 laiteries. Ses résultats sont consignés au IIe volume du Congrès d'Hygiène alimentaire de Bruxelles, 1910, page 227. Seuls furent publiés les résultats de 37 laiteries sur les 80 ; on eut soin de choisir les laiteries du pays plat, là où les indices A.V.S. sont plus élevés qu'en haute Belgique.

En cachant certains résultats, le rédacteur du mémoire, M. GroGNARD, voulait-il démontrer que nos beurres belges échappaient à l'injuste réprobation qui frappait à cette époque les "anormaux "? ?'après lui, un peu plus de $1 \%$ des beurres titraient de 26 à 27 A.V.S., $1 / 2 \%$ de 25 à $26,1 \%$ atteignaient presque 25 !

Ces conclusions étaient fausses, et il est heureux que M. I. WAUters, docteur ès sciences, ait pu consulter l'ensemble des documents et rétablir ainsi la vérité : nous avions en Belgique, comme dans d'autres pays, notre large part de beurres dits anormaux! (même congrès, p, 181).

Le mémoire officiel affirmait, de plus, que, pour une laiterie déterminée, les valeurs A.V.S. traduites, au cours de deux années, 
dans un graphique, étaient “à peu près identiques et se superposaient ». Il ne s'agissait que de petites et de moyennes laiteries; or, si l'on prenait la peine de consulter les graphiques, on constatait que la superposition était exceptionnelle, car d'une année à l'autre les A.V.S. variaient sensiblement pour une même laiterie.

On pourra contrôler plus loin les variations présentées d'une année à l'autre par le graphique des beurres de la Frise hollandaise, là où se trouvent représentés des millions de kilogrammes.

Cet exemple illustre bien la mentalité d'alors dans certains milieux : le fait d'avoir des beurres à indices faibles passait pour une tare nationale!

$$
* * *
$$

La première raison donnée pour expliquer la déficience en A.V.S. des beurres, c'est qu'ils provenaient de vaches mal alimentées ou malades; on repoussait aussi énergiquement l'idée qu'un chiffre aussi bas que 25 ait pu être donné par un troupeau important, il ne pouvait provenir que d'une ou deux unités; leurs earactères déficients se fondaient alors dans la masse qui, elle, devait avoir une composition normale.

Et, c'est de cette époque que date le qualificatif beurre normal ; par déduction logique, le terme "beurre anormal" fut appliqué au produit ne répondant pas aux normes fixées. Ce mot "anormal " souleva des protestations bien légitimes, car, il était, en effet, injuste d'affubler un produit pur et naturel d'une étiquette aussi péjorative.

A-t-on jamais songé à appliquer le même qualificatif au lait renfermant $2,8 \%$ de matière grasse, alors que la teneur ordinaire est de $3 \%$ et plus ? Et cependant, ici, il y a déficit de matière grasse.

Le Dr Swaving a très bien caractérisé la mentalité de certains milieux d'alors en écrivant "le beurre anormal est le souffredouleur de notre impuissance scientifique » et le ministre de l'Agriculture de l'époque ajoutait : "C'est le zondenbok de la science" (le boue chargé des péchés d'Israël)!

LAHACHE et MARRE. dans leur livre Beurre de vache et graisse de coco (Paris, 1912, Maloine, éditeur), proclamaient : "Nous estimons que les beurres auxquels on se plaît à accoler le qualificatif d'anormal, ne méritent pas d'être livrés à la consommation, et nous demandons pour eux un régíme qui leur retire désormais le droit d'être vendus comme aliments. " Nous trouvons des opinions plus récentes (Le Lait, 1933) exprimées comme suit : "Les indices R.M. (A.V.S.) sont faibles lorsque les vaches sont réduites à un état de misère physiologique due à une stabulation exagérée. » 
Les beurres à indices faibles sont moins digestibles et se rapprochent beaucoup de la margarine. " "Il ne convient pas de reconnaître aux beurres d'indices faibles une valeur marchande pas plus que ne l'ont reconnu CouRdon et Rousseaux en 1901. "Les beurres à indices faibles sont moins digestibles, moins vitaminisés. 》

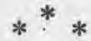

Quand le beurre était-il "anormal " ?

Pour certains experts le chiffre 28 A.V.S. marque la norme de pureté en dessous de laquelle tout beurre est anormal, d'autres ont fixé le chiffre 26 , et certains consentent même à descendre de 2 ou 3 unités s'il est établi que le beurre analysé provient de quelques vaches (2 à 5 ). L'accord unanime sur un chiffre déterminé n'a jamais été réalisé.

Mais, si l'on examine, en se plaçant au point de vue de la composition centésimale du beurre, ce que représentent ces quelques unités d'A.V.S., on doit reconnaître leur importance minime. En effet, on admet que la norme 28 représente approximativement les $7 \%$ des glycérides des acides du groupe butyrique, chaque unité A.V.S. vaut done $7 / 28 \mathrm{e}$, soit $0,25 \%$; un beurre titrant 25 A.V.S. ne contiendrait que 6,25 de butyrine au lieu de 7 , et si même son indice descendait à 20 , son taux de butyrine serait de 5 au lieu de 7 . Nous verrons plus loin combien cette modification est de minime importance à côté de celles que nous révèlent les indices d'iode, de HeHner, de Kottstorfer et surtout des indices de réfraction (A.Z.).

$$
\text { ** } *
$$

L'indice d'iode, qui totalise l'ensemble des glycérides non saturés, varie pour les beurres de 30 à 50 , pour le groupe des oléo et du neutral de 70 à 88 , et pour le groupe des palmistes (coco) de 10 à 17. Les indices de saponification des trois groupes montrent également des variations accusées pour chacun de ces groupes, mais les écarts les plus impressionnants sont ceux des indices de réfraction (A.Z.). Ces indices ne nous donnent, il est vrai, aucune indication d'ordre chimique, ils se bornent à une simple notation physique, laquelle, prise en elle-même, nous montre les changements que la composition du beurre a subis, sans nous en indiquer la nature.

Les glycérides du groupe des palmistes ont un indice A.Z. voisins de 35,5 , ceux du groupe oléo 49,5 , et ces valeurs ne varient guère. On admet que l'acide laurique et ses congénères forment la base de la cocose et de la palmitine, et que les acides oléique, stéarique et palmitique prédominent dans l'oléo et le neutral. 
Ces matières grasses sont, on le sait, à l'opposé l'une de l'autre sì on considère leurs indices chimiques.

Les limites extrêmes des indices A.Z. des beurres ont été de 38 et 47 . Ces deux chiffres sont bien voisins, l'un de la cocose, l'autre de l'oléo.

Quelle ne doit pas être la différence de la composition chimique de deux beurres présentant l'un 38 A.Z., l'autre 47 ?

Supposons maintenant que nous possédions un procédé nous permettant de doser exactement l'acide stéarique, dans le complexe beurre. Quel serait le pourcentage des stéarines dans un beurre à 38 A.Z. ? Quel en serait le pourcentage dans un beurre à 47 A.Z. ?

Ce pourcentage, nous le devinons, serait d'autre ordre de grandeur que celui représenté par la totalité des dérivés de l'acide butyrique, soit $7 \%$. Et ce serait peut-être ici le cas d'appliquer l'épithète si malencontreuse de beurre "anormal ".

$$
*^{*} *
$$

Rapprochons les valeurs A.V.S. et A.Z. et voyons si, entre elles, il existe un rapport quelconque.

Les stations de contrôle de l'Etat hollandais nous apportent sur ce point des documents de première valeur. Elles déterminent en effet toutes les semaines ou tous les quinze jours, les indices A.V.S. et A.Z. de toutes les laiteries; la station principale de Leyde centralise les résultats. Ceux-ci doivent, à l'heure actuelle, dépasser le million. En 1909, ils étaient au nombre de 91.077, qui sont réunis en un tableau publié par les Annales des Falsifications et des Fraudes (janvier 1909, n० 3 , pages 14 et 15 .).

Examinons ${ }^{\bullet}$ ce tableau en mettant en regard d'un chiffre déterminé d'A.V.S. les valeurs réfractométriques différentes présentées par ces beurres. Prenons le chiffre 28 A.V.S. atteint par 16.642 échantillons. Le chiffre A.Z. le plus fréquent est 44 (7.746 beurres), le moins élevé est de 41 ( 86 beurres), le plus élevé de 46 ( 175 beurres).

Voici la répartition complète des résultats donnés pour les beurres titrant 28 A.V.S.

Sur les 16.642 échantillons :

86 marquaient 41 A.Z.

\begin{tabular}{|c|c|c|}
\hline 1.280 & 1 & 42 \\
\hline 3.585 & " & 43 \\
\hline 7.746 & " & 44 \\
\hline 3.770 & ") & 45 \\
\hline 175 & ” & 46 \\
\hline
\end{tabular}

$\mathrm{Si}$, maintenant, je reprends pour les autres valeurs A.V.S. le chiffre A.Z. le plus souvent atteint, je puis établir le tableau suivant : 
30/31 A.V.S. correspond à 42 A.Z.

\begin{tabular}{|c|c|c|c|}
\hline 29 & 》) & ) & 43 \\
\hline 28 & 》) & \#) & 44 \\
\hline 27 & ") & 》 & 45 \\
\hline 26 & ” & 》) & 45 \\
\hline 25 & 》) & ) & 45 \\
\hline 24 & 》) & 》) & 45 \\
\hline 23 & 》) & ") & 46 \\
\hline 22 & 》) & ) & 46 \\
\hline 21 & ) & 》 & 46 \\
\hline 20 & ) & ) & 46 \\
\hline 19 & ” & 》 & 47 \\
\hline 18 & 》 & D & 47 \\
\hline
\end{tabular}

Ces chiffres ne doivent pas être considérés comme des moyennes, ils n'en présentent pas d'ailleurs le caractère, ils ont été extraits du tableau là où ils se sont le plus souvent rencontrés. Si, maintenant, nous prenons comme base non plus l'indice A.V.S., mais l'indice A.Z., nous constatons que pour le chiffre 44 , les A.V.S. ont varié de 22 à 34 .

Que déduire de la composition centésimale d'un produit qui, pour un même indice 28 , peut présenter une réfraction qui va de 41 à 46 , ou qui, pour une réfraction de 44 , peut varier en A.V.S. de 22 à 34 ?

Quel est l'auteur qui a écrit : "La détermination de la composition centésimale du beurre, à supposer que nous possédions des méthodes exactes de séparation, se heurtera toujours aux différences profondes de composition du produit. »

Il y a 150 ans que l'illustre chimiste français CHEvREUL écrivait : "Le beurre est un composé de différents glycérides. "

Définition fort prudente, mais qui me paraît la seule exacte à l'heure présente.

$$
*^{*} *
$$

Voyons maintenant les résultats de différentes enquêtes entreprises pour établir les variations d'un beurre déterminé au cours d'une année. On comprend que j'écarte systématiquement les très nombreuses études faites pour déterminer l'influence de l'alimentation. Un aliment, tel les collets de betteraves, cela est acquis, augmente la quantité d'A.V.S. ; mais l'alimentation par les betteraves est forcément limitée par le temps; on les utilise durant 15 jours ou un mois; or, nous étudions ici des variations annuelles et non mensuelles.

Nous ne pouvons plus nous appuyer sur les expériences faites 
sur 1, 2 ou 3 unités, ces expériences fussent-elles continuées durant toute l'année.

Je dois également négliger les résultats des enquêtes officielles faites en Belgique pour les raisons que j'ai exposées plus haut. Je citerai done les résultats obtenus par MM. VAN ENGELen et P. WaUters portant sur la production de 12 bêtes; ceux du Professeur LAXA (21 laiteries de la Moravie; le nombre de bêtes n'est pas mentionné); les miens au cours de deux enquêtes portant sur des troupeaux de 15 à 20 bêtes.

On retiendra les études très complètes et très intéressantes du Professeur Polonowski et de Mile $D^{r}$ Thomas, portant sur la production de quatre centres de $6,10,12$ et 12 vaches.

Enfin, les statistiques des contrôles hollandais et de leur centre Leyde nous apporteront des documents de première valeur. Le contrôle de La Frise porte sur une production annuelle de 18 à 23.000.000 de kilogrammes (chiffres des dix dernières années). Les contrôles du Limbourg, de Dreuthe, du Brabant méridional, analysent maintenant et chaque année 5.000.000 de kilogrammes environ; ce chiffre est approximatif, mais voisin de la vérité.

La liste qui suit mentionne les mois durant lesquels les indices les plus bas et les plus élevés ont été constatés, ainsi que l'indication de ces indices (A.V.S.) :

Beurres de Bouchout (VAN Engelen et Wauters). - Août : 23 - Février : 34.

Beurres de Moravie (LAxA). - 21 laiteries. - Mars : 24 Juin : 32,2 .

Beurres de Herve ( $1^{\text {re }}$ enquête Hотом) 3 fermes. - Décembre : 20 à 21 - Mars, avril, mai : 33 à 36 .

Beurre de Herve ( $2^{e}$ enquête Hoton) 2 fermes. - Novembre : 22,5 - Mars et avril : 35 et 37,4 .

Beurre danois (origine ?, contrôlé), - Août : 30 - Avril : 33.

Beurres du Nord de la France (Polonowski et Thomas). Steenwoorde. - Juillet : 20 - Février : 32,2.

Palempin. - Juin : 21,9 - Février : 32.

Vendegnies. - Octobre : 18,3 - Décembre : 32,4.

Winezeele. - Octobre : 15 - Décembre : 32,5 .

On peut situer en avril et octobre pour tous les contrôles hollandais les époques où les beurres présentent les maxima et minima d'A.V.S. - le contrôle du Brabant méridional (Linhoven) a fait cependant exception à cette règle en 1913.

La station de la Frise (Leewarden) est la plus importante du pays ; elle contrôle de 1927 à 1938 des quantités de beurre qui ont varié entre 18 et 23 millions de kilogrammes provenant de 104 laite- 
ries agréées. Durant ces onze années, les minima et maxima ont été généralement atteints entre le 15 mars-1 er avril, et octobre-15 novembre. Les maxima ont varié, durant cette période, entre 31,5 et 32,5 ; les minima de 25,5 à 27,5 . On aurait tort de croire que toutes les laiteries du contrôle Frison fournissent des produits qui ne s'écartent guère de ces limites. C'est ainsi que je relève fin octobre 1938 des écarts sérieux entre deux laiteries; l'une d'elles a fourni un beurre titrant A.V.S. 22,4, A.Z. 45,3, l'autre A.V.S. 28,3, A.Z. 42,8. Il s'agit ici, qu'on ne l'oublie pas, de deux laiteries d'une même province (même climat, même race, même alimentation). Chaque année, la Station de contrôle de la Frise publie le rapport des travaux de son laboratoire, dirigé par l'ingénieur-chimiste M. VAN BALEN-WALter. L'intéressante brochure mentionne les chiffres A.V.S., A.Z., ainsi que le pourcentage en eau des beurres analysés. Le nombre d'analyses a été en 1938 de 5.824. Un tableau indique les chiffres maxima et minima ainsi que les moyennes; de plus un graphique, reproduit ci-après, montre les variations bimensuelles de composition des beurres.

Si on transcrit en graphiques les résultats des enquêtes faites ailleurs qu'en Hollande, on observe, à de très minimes exceptions, que le relèvement des A.V.S. commence habituellement dans la quinzaine où les beurres ont atteint le taux le plus bas. De même qu'environ 15 jours après que le chiffre le plus élevé a été atteint, la descente commence.

A cet égard, les graphiques des onze dernières années de la Station de Leewarden sont caractéristiques; je n'y note qu'une seule exception digne d'être relevée, et on la trouve précisément ci-contre : du $1^{\text {er }}$ mars au $1^{\text {er }}$ mai 1938 , le chiffre 31,5 A.V.S. n'a guère varié ; ce n'est que le $1^{\text {er }}$ juin que la descente est nette $(30,5)$.

Un fait qu'on peut observer chaque année, e'est que de janvier à mai les indices de réfraction restent figés près de 42,5 , alors que la montée des A.V.S.' commencée vers octobre se poursuit à un rythme régulier jusqu'en mars. L'écart de janvier à mars n'est pas énorme (29 à 31,5), mais n'oublions pas qu'il marque les indices moyens de 20 millions de kilogrammes et, à ce titre, il acquiert une grande importance.

$$
\text { *** }
$$

Peut-on soutenir, après avoir vu ces graphiques, que les conditions générales de la vie du bétail diffèrent d'avril à juin ? Ne tendent-elles pas, à cause de l'abondance des fourrages et de l'amélioration de la température, à être meilleures? Comment expliquer alors l'abaissement des A.V.S. qui va se poursuivre régulièrement jusqu'en octobre-novembre? 
INDIGES MOYENS DES A. V. S. DÉTERMINÉS TOUS LES 15 JOURS

.m............. du 1er Janvier 1937 au 1er Janvier 1938

du 1er Janvier 1938 au 1er Janvier 1939

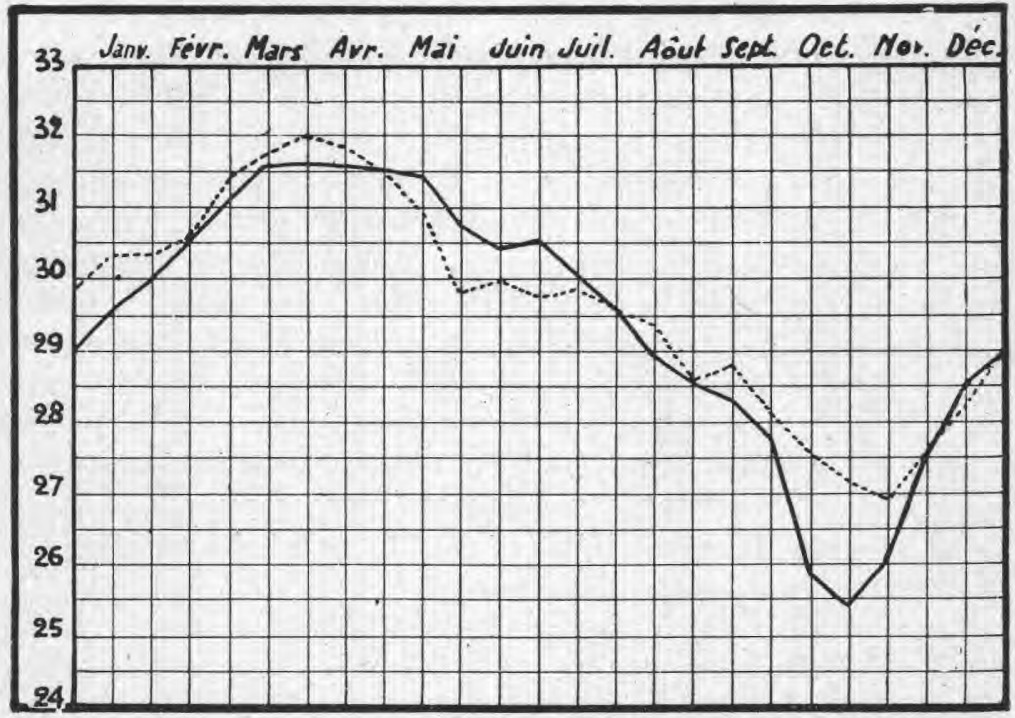

INDIGES MOYENS REFRACTOMETRIOUES

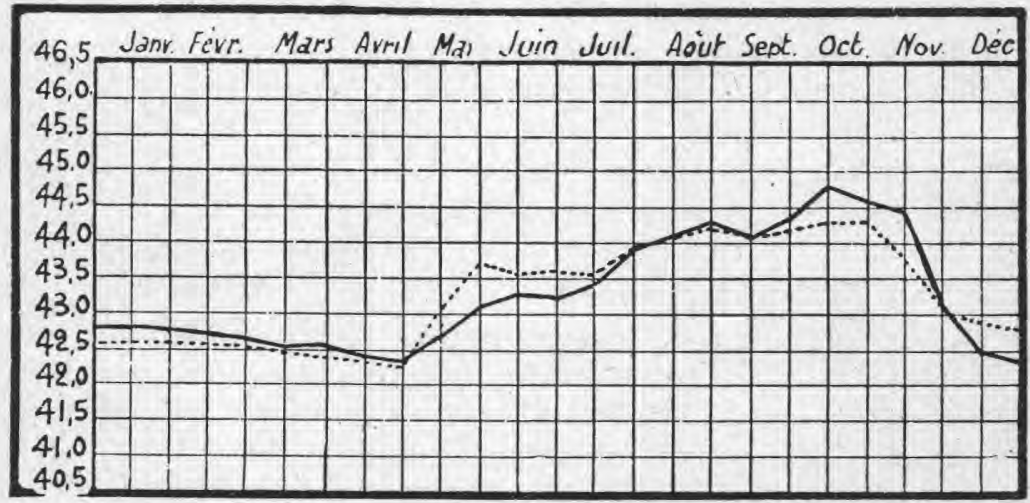

Et, si nous comparons la situation durant ces derniers mois avec celle de décembre et jusqu'à la mise en pâture, nous devons reconnaître que les conditions d'existence du bétail ne sont pas meilleures, au contraire. Comment expliquer ainsi le relèvement des A.V.S.

Est-il possible, après examen des graphiques et connaissant la manière d'élever le bétail en Frise, de maintenir l'hypothèse suivant laquelle les beurres à faibles indices A.V.S. sont dus à une mauvaise hygiène, à une alimentation déficiente ou défectueuse, aux conditions 
atmosphériques du moment ? La production beurrière frisonne jouit d'une réputation mondiale, et elle est méritée.

Avouons notre impuissance à expliquer ce fait brutal devant lequel nous devons nous incliner; ce n'est pas en déclarant que les beurres titrant moins de 26 seront qualifiés d'anormaux que nous aurons résolu la question. N'imitons pas les médecins qui, ne pouvant expliquer le rhume de cerveau, l'ont baptisé "Coryza»! MM. VAN ENGELEN et P. WAUTERs terminaient leur enquête en 1899 par ces considérations qui sont encore vraies aujourd'hui : "Ces beurres (anormaux) peuvent se rencontrer en toutes saisons, ils ne sont dus ni au régime alimentaire ni au temps plus ou moins écoulé depuis l'époque du vêlage. Ils doivent se produire sous l'influence de conditions physiologiques que nous n'avons pu déterminer. ")

$$
\text { *** }
$$

Faut-il apporter un argument de plus pour montrer l'inexactitude des explications relatives à la production des beurres à faibles indices A.V.S. ?

Je le trouve dans la comparaison des beurres des contrôles du Limbourg et de la province de Drenthe. Les beurres de Limbourg sont toujours riches en A.V.S., ceux de la Drenthe sont pauvres. Si on m'oppose la distance (environ $150 \mathrm{~km}$.) qui sépare les deux contrôles, je dirai que la Frise est proche de Drenthe, que le climat y est sensiblement le même, et qu'en Frise, enfin, la richesse des beurres en A.V.S. est très voisine de celle des beurres du Limbourg. Dans ces provinces, nous avons une même race de bétail, les soins y sont les mêmes, la nourriture ne diffère guère, la rentrée du bétail à l'étable est peut-être un peu avancée en Frise. Les deux tableaux suivants ont trait à l'année 1912 (Annales des Falsifications, août 1913, L. НотоN). Ils sont significatifs.

En prenant comme base non plus 28 A.V.S., mais 25, voici le tableau caractéristique de l'année 1912 : la $1^{\text {re }}$ colonne comprend le nombre total des beurres des laiteries, le $2^{\text {e }}$ le nombre de beurres titrant moins de 25 , la seconde celle des beurres titrant plus de 30 dans les deux contrôles.

Un second tableau nous donne, mais seulement pour les deux mois essentiels, mai et novembre, les mêmes résultats pour les sept années antérieures à 1913.

Les quantités des beurres contrôlés de ces deux régions sont sensiblement les mêmes, elles étaient pour l'année 1912 de 3.450 .000 kilogrammes en Drenthe (pour 70 laiteries) et de 3.900.000 kilogrammes en Limbourg (pour 85 laiteries). Elles ont augmenté depuis. 


\begin{tabular}{|c|c|c|c|c|c|c|}
\hline \multirow{2}{*}{ 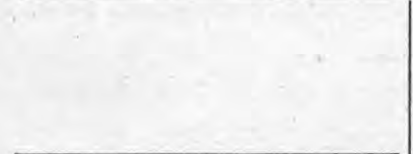 } & \multicolumn{3}{|c|}{ Contrôle du Limbourg } & \multicolumn{3}{|c|}{ Contrôle de Drenthe } \\
\hline & Totaux & $\begin{array}{l}\text { Moins } \\
\text { de } 25\end{array}$ & $\begin{array}{l}\text { Plus } \\
\text { de } 30\end{array}$ & Totaux & $\begin{array}{l}\text { Moins } \\
\text { de } 25\end{array}$ & $\begin{array}{l}\text { Plus } \\
\text { de } 30\end{array}$ \\
\hline Janvier ............... & 259 & 1 & 100 & 135 & 8 & 0 \\
\hline Fóvrier $\ldots \ldots \ldots \ldots \ldots$ & 253 & 1 & 90 & 135 & 1 & 2 \\
\hline Mars $\ldots \ldots \ldots \ldots \ldots \ldots$ & 251 & 0 . & 187 & 140 & 0 & 9 \\
\hline Avril $\ldots \ldots \ldots \ldots \ldots \ldots$ & 235 & 0 & 176 & 133 & 0 & 18 \\
\hline Mai $\ldots \ldots \ldots \ldots \ldots \ldots$ & 245 & 0 & 42 & 132 & 0 & 25 \\
\hline Juin $\ldots \ldots \ldots \ldots \ldots \ldots$ & 240 & 0 & 32 & 132 & 0 & 1 \\
\hline Juillet . ............. & 239 & 0 & 24 & 132 & 25 & 0 \\
\hline Août $\ldots \ldots \ldots \ldots \ldots \ldots \ldots$ & 239 & 1 & 0 & 134 & 10 & 0 \\
\hline Septembre ............ & 240 & 1 & 30 & 138 & 24 & 0 \\
\hline Octobre $\ldots \ldots \ldots \ldots \ldots$ & 236 & 0 & 63 & 145 & 0 & 0 \\
\hline Novembre.............. & 233 & 0 & 109 & 133 & 20 & 0 \\
\hline \multirow[t]{3}{*}{ Décembre $\ldots \ldots \ldots \ldots \ldots$} & 236 & 0 & 8 & 154 & 9 & 0 \\
\hline & \multicolumn{3}{|c|}{ Contrôle de Drenthe } & \multicolumn{3}{|c|}{ Contrôle du Limbourg } \\
\hline & Totaux & $\begin{array}{l}\text { Moins } \\
\text { de } 25\end{array}$ & $\begin{array}{l}\text { Plus } \\
\text { de } 30\end{array}$ & Totaux & $\begin{array}{l}\text { Moins } \\
\text { de } 25\end{array}$ & $\begin{array}{l}\text { Plus } \\
\text { de } 30\end{array}$ \\
\hline \multicolumn{7}{|l|}{$1906:$} \\
\hline Mai $\ldots \ldots \ldots \ldots \ldots$ & 149 & 1 & 10 & 700 & 0 & 274 \\
\hline Novembre.......... & 151 & 61 & 0 & 716 & 0 & 195 \\
\hline \multicolumn{7}{|l|}{ 1907: } \\
\hline Mai $\ldots \ldots \ldots \ldots \ldots$ & 177 & 0 & 20 & 678 & 0 & 444 \\
\hline Novembre.......... & 174 & 39 & 0 & 627 & 2 & 222 \\
\hline \multicolumn{7}{|l|}{$1908:$} \\
\hline Mai $\ldots \ldots \ldots \ldots \ldots$ & 157 & 0 & 16 & 336 & 0 & 126 \\
\hline Novembre........... & 160 & 36 & 0 & 346 & 0 & 114 \\
\hline \multicolumn{7}{|l|}{$1909:$} \\
\hline Mai $\ldots \ldots \ldots \ldots \ldots$ & -154 & 0 & 5 & 281 & 0 & 209 \\
\hline Novembre........... & 147 & 64 & 0 & 290 & 1 & 22 \\
\hline \multicolumn{7}{|l|}{ 1910: } \\
\hline Mai $\ldots \ldots \ldots \ldots \ldots$ & 147 & 0 & 21 & 286 & 0 & 204 \\
\hline Novembre........... & 145 & 30 & 0 & 291 & 0 & 24 \\
\hline \multicolumn{7}{|l|}{ 1911: } \\
\hline Mai $\ldots \ldots \ldots \ldots \ldots$ & 142 & 0 & 14 & 263 & 0 & 178 \\
\hline Novembre.......... & 133 & 43 & 0 & 262 & 0 & 36 \\
\hline \multicolumn{7}{|l|}{ 1912: } \\
\hline Mai $\ldots \ldots \ldots \ldots \ldots$ & 132 & $\mathbf{0}$ & 25 & 245 & 0 & 42 \\
\hline Novembre........... & 133 & 20 & 0 & 233 & 0 & 109 \\
\hline
\end{tabular}

Quelle explication donner à ces chiffres ? 
Combien le Dr BaLavoine a-t-il raison de déclarer (voir le début de cette étude): "Que les motifs pour justifier l'ostracisme contre les beurres "anormaux " ne résistent pas à l'examen!"

On va jusqu'à attribuer à ces parias des qualités moins digestives qu'aux normaux! Faut-il s'attarder à réfuter cette imputation ? (1). Mais, je tiendrais à rencontrer ceux qui affirment que les beurres renferment les vitamines $\mathrm{A}$ et $\mathrm{D}$, eette dernière durant seulement les mois d'été; or, ce n'est qu'en été qu'on trouve les beurres à faibles indices A.V.S.

$$
* * *
$$

Ce qualificatif « anormal » est faux, antiscientifique et péjoratif.

La législation belge (loi du 10 août 1900 et arrêté du 31 octobre 1900 , art. 3) l'a admis durant 4 ans, puis l'a remplacé par "les beurres ayant des caractères anormaux") (arrêté du 21 novembre 1904), mais il est à noter que leur vente n'était pas interdite si un contrôle officiel établissait leur pureté (2-3). La législation actuelle est muette sur cette question qui a fait couler tant d'encre, le mot " anormal " n'y figure plus. Supprimons done ce vocable de notre littérature.

Liége, avril 1940.

(1) En 1910 et durant les années suivantes, nombreux étaient les médecins qui défendaient l'usage du beurre aux diabétiques et aux albuminuriques, et ce à cause de la butyrine ! Ils étaient soumis au régime de la margarine.

(2) Arrêté royal du 31 octobre 1900 :

On entend par beurres anormaux ceux dont les caractères, sans révéler avec certitude la falsification ou l'altération grave, s'écartent de ceux de la généralité des beurres purs.

Pour être déclarés anormaux ils doivent présenter au moins deux des caractères suivants :

Un indice A.Z. à $40^{\circ}$ supérieur à 46 .

Un indice Crismer supérieur à 59 .

Une densité inférieure à 864 .

Un indice d'A.V.S. inférieur à 25.

Un indice Hehner supérieur à 89,5 .

Un indice de Kottstorfer inférieur à 221.

(3) L'arrêté royal du 21 novembre 1904 défend la vente des beurres ayant un caractère anormal, à moins qu'un contrôle officiel de leur production n'ait été effectué. Sont déclarés a beurres à caractères anormaux » ceux qui, outre un indice A.V.S. inférieur à 28, présentent l'un ou l'autre des earactères suivants :

Un indice A.Z. à $40^{\circ}$ supérieur à 44 ,

Un indice Crismer supérieur à 57.

Une densité inférieure à $\mathbf{8 6 5}$.

Un indice Hehner supérieur à 88,5.

Un indice de Kottstorfer inférieur à 222. 


\title{
BIBLIOGRAPHIE
}

LAHACHe et MARRe. Beurre de vache et graisse de coco. Editeur : Maloine, Paris.

E. Grognard. $2^{\mathrm{e}}$ Congrès d'Hygiène alimentaire, Bruxelles $1910,2^{\mathrm{e}}$ volume, p. 227.

I. Wauters. $2^{\text {e }}$ Congrès d'Hygiène alimentaire, Bruxelles, 1910. $2^{e}$ volume, p. 181.

Van Engelen et P. Wauters. Enquête sur les beurres de Bouchout. Bull. de l'Agriculture belge, 1899.

Dr Swaving. Le Lait, XIII, mars 1933.

$-2^{\mathrm{e}}$ Congrès de Laiterie, Bruxelles, $1^{\text {re }}$ Section.

LAXA. Annales des Falsifications, 1932, p. 529.

Prof. Polonowski et Dr Thomas. Le Lait, XIII, mars, avril, mai 1933, pp. $249,466,583$.

Dr Balavoine. Le Lait, XIX, décembre 1939, p. 1027.

Dr Van Beneden et Prof. Vivario. Le Lait, XVI, février 1936, p. 113.

Hотом. Beurres anormaux. Journal de Pharmacie d'Anvers, 1904.

- Beurres purs, beurres falsifiés. Journal de Pharmacie d'Anvers, 1906.

- Les indices de réfraction. Annales des Falsifications, janvier 1909.

- Beurres du pays de Herve (1re ótude). Journal de Pharmacie de Belgique ; $2{ }^{\circ}$ étude. Journal de Pharmacie de Belgique, 1930, nos 22-24 et 36; 25-24.

- Glycérides de beurre. Journal de Pharmacie de Belgique, 1932, no 33.

- Beurres anormaux. Annales des Falsifications, août 1913.

\section{L'UTILISATION DU SÉRUM}

\author{
par \\ G. GENIN \\ Ingénieur E. P. C.
}

On sait que l'utilisation industrielle des sous-produits de l'industrie laitière est liée à un certain nombre de facteurs économiques qui ont trait à la nature des produits que l'on peut isoler, aux dépenses qu'entraîne la séparation de ces produits et aux cours auxquels on peut les revendre. Lorsqu'on utilise dans une laiterie du lait entier en vue de le transformer en produits marchands, on admet qu'on peut en tirer environ $10 \%$ de crème, $2,8 \%$ de caséine et $75 \%$ de sérum. Ce sérum est essentiellement constitué d'eau, de lactose, de lactalbumine et de vitamine G. La méthode la plus simple pour la récupération de ce sérum est de le concentrer de façon à obtenir un liquide épais que l'on mélange avec de la poudre de sérum desséché, de façon à obtenir une substance de consistance granulaire qui est à son tour desséchée et pulvérisée. Cette poudre, qui est la poudre de sérum du commerce, est essentiellement utilisée pour la nourriture des animaux et de la volaille. Malheureusement, le prix de vente de cette poudre est très faible et il est rare que la récupération de 